\title{
Towards Pervasive Motor and Cognitive Rehabilitation Strategies Mediated by Social Interaction
}

\author{
Hoang H. Le, Martin J. Loomes, and Rui C. V. Loureiro
}

\begin{abstract}
This paper introduces the concept of socially mediated interventions targeting specific deficits anywhere, in public spaces beyond the hospital/clinical context. Such concept would provide affordable and engaging experiences carefully designed to promote social interaction and affordable pervasive interventions without the 'rehabilitation tag' associated. Several playful group activities supported by haptic robots have been conducted to determine how people perceive this type of approach. This paper presents a set of playful group activities piloted to start testing the feasibility of the concept.
\end{abstract}

\section{INTRODUCTION}

$\mathrm{T}_{\mathrm{r}}$ He assistive and rehabilitation robotics field has grown remarkably in the past decade. Technologies have been developed to provide intensive training and enhanced tools for patient management. Historically, assistive robotics has taken more of a role of physical assistance with movements. Recently, a new robotic field called socially assistive robotics has arisen to support social therapy which has the potential to benefit not only people with cognitive and learning disabilities such as individuals with autism but also individuals with physical disabilities.

Post-stroke patients might be returning home sooner than in the past due to the shortening of hospital stays [1]. Consequently, there is an increased need to continue their rehabilitation at home. In order to do so, robot-aided systems that allow the remote management of therapies could be a solution. Such systems should not only reduce the care burden imposed on the patient's family but also facilitate access to therapy on demand when unable to access the rehabilitation centre. The key issue however, is to come up with systems that engage the patient in the therapy regime by maintaining motivation and attention levels that might otherwise be lost due to isolation and lack of interaction with other people, patients and therapists. The 'fun' factor and feeling of 'accomplishment' in this context is also crucial for rehabilitation simply because it might enable patients to practice more in an engaging task.

Understanding the individual's goals is important as needs and motives often drive goals, influence behaviors and affect personal effort. Such needs might surface out of emotional,

H. H. Le and M. J. Loomes are with the School of Science and Technology of Middlesex University, The Burroughs, Hendon, London, NW4 4BT, UK (e-mail: h.le@mdx.ac.uk or m.loomes@mdx.ac.uk).

R. C. V. Loureiro is with the Aspire Centre for Rehabilitation Engineering and Assistive Technology, University College London, Royal National Orthopaedic Hospital, Brockley Hill, Stanmore, Middlesex, London, HA7 4LP, UK (e-mail: r.loureiro@ucl.ac.uk). independence, and social goals that may be obstructed as a result of neurological impairment. Thus the understanding of how humans interact (collaborate, compete, cooperate) with each other (behaviors) is critical to both motor learning and rehabilitation. Strategies for increasing and maintaining patient interest vary. Some systems incorporate activities of the daily living, others attempt to motivate patients via functional reaching and grasping; others address the social exclusion problem by developing systems that provide cooperative tasks between patient and therapist and through collaborative play between individual patients and between patient groups [2].

Cognitive impairment combined with motor impairment is doubly isolating. Some evidence suggests that tele counselling can have a positive impact on managing issues such as sleep difficulties, pain, anxiety and depression. Children with disability are often isolated and the resources for their care very limited. Children with autism for example, usually have difficulties with social and communication abilities hence they may depend on specialist support throughout their lives. It is believed that early intervention through playful activity may be effective in improving social communication and interaction skills [3]. It is safe to say that autistic children have a similar need as for example post-stroke patients: a better assistive tool that enables them to have intensive treatment at home or other social environments outside of the clinic. As a result, they have the same challenge as post-stroke patients; to maintain motivation to follow therapeutic exercises.

\section{Pervasive Rehabilitation}

\section{A. Concept}

The idea behind pervasive rehabilitation is to explore further the concept of group interaction with possible benefits of engaging with artifacts, such as paintings, music/soundscapes and creative expression or playful activity beyond the clinic or home. These experiences when mediated by theranostic technologies, which promote physical exploration through aural and sensory cues, could have a positive effect on rehabilitation and cognitive development. Our long-term goal is to combine different sensing and robotic technologies within a unifying software framework to provide affordable therapy on-demand, executed in a fun collaborative way by children and family members, carers and support networks. 


\section{B. Pilot group activities}

We have piloted a few group activities to start testing the feasibility of the concept.

1) Group interaction with a specific goal

- Eight healthy participants (age $23 \pm 7$ years) were recruited to work as a group to make a robot play a bell (fig. 1). Participants were given 10 minutes to achieve the task and informed that the sensor (Microsoft Kinect) next to the robot would track their movements but were not told the sensor would track only one participant at each time [4].

- Twenty-four healthy participants (age $40 \pm 20$ years) worked as pairs in a shared virtual environment. They were instructed to collaborate with each other in order to lift a virtual cube (using a stylus-based haptic device providing appropriate haptic feedback) and placing it on the top of another cube (fig. 2).

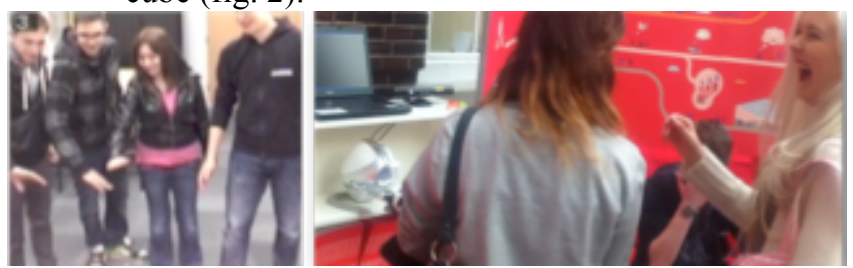

Fig. 1: Social activity mediated by a robotic bell.

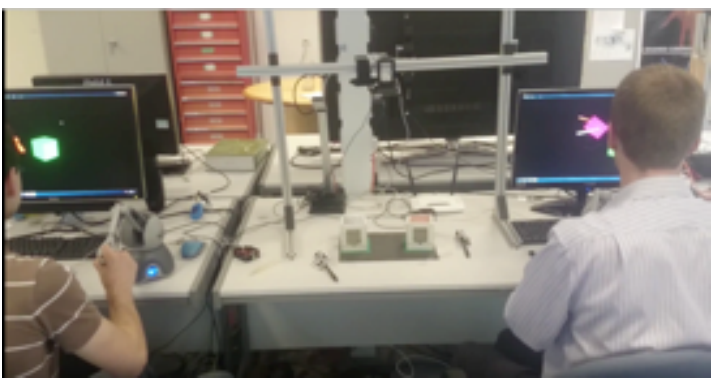

Fig. 2: Collaborative virtual 'stacking cubes' activity.

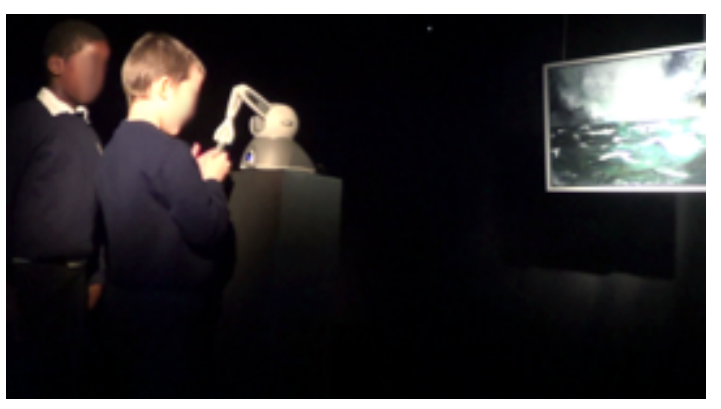

Fig. 3: Explorative activity with an interactive painting.

2) Group interaction without any goal (exploration)

Seven autistic children (all male, aged 7 y/o \pm 14 months) diagnosed with sever general learning disabilities were asked to explore an interactive painting (with haptic and sound feedback closely mapped to the painting physical elements) using Phantom Omni as long as they want to (fig. 3) the setting was in a real exhibition gallery and following a period of interaction with the painting on their own, other autistic children were introduced with the aim of facilitating interaction among the children. [5].

\section{RESULTS AND DISCUSSION}

The results obtained with the pilot group activities are very promising. Participants enjoyed their activities and made a lot of movements (on average 3,500 movements for the robotic bell group). They also interacted with their partner(s) performing together the task, which is very important especially for cognitive impairment. Although autistic children made varied movements and some participants seemed to be more explorative than others, the fact that they were inquisitive as to where the sound of the water and touch sensation came from, it prompted for conversation and to taking turns to use the haptic device in the interactive painting activity. This is interesting to observe because according to their teacher, they usually do not interact to each other.

Verbal communication was crucial to successfully complete the activities with a specific goal. Participants had to discuss and to come up with a consensus strategy to fulfill the goal and complete the activity. In the virtual environment (Fig. 2), the only way for participants to achieve the goal (stack cube on top of another one) was to keep talking and instruct each other. Hence this type of interaction has potential to encourage communication thus improving social interaction skills.

\section{CONCLUSION}

By empowering people to engage with therapeutic interventions in a fun way, outside the clinic and home would enable families and care networks to take an active role on the rehabilitation process.

We aim to develop the understanding necessary to evolve the concept into a pervasive technology which, will lead in the future to a wealth of possible interaction principles - any part of the body could be used to 'play' with an interactive installation from the head to the feet and target different physical and cognitive deficits.

\section{REFERENCES}

[1] M.K. Holden, T.A. Dyar, L. Schwamm, and E. Bizzi. Virtual Environment- Based Telerehabilitation in Patients with Stroke. Teleoperators and Virtual Environments 2005 14:2, 214-233.

[2] Andrade, A.O., Pereira, A.A., Walter, S., Almeida, R., Loureiro, R.C.V., Campagna, D., Kyberd, P.J. (2014). Bridging the Gap Between Robotic Technology and Health Care. Biomedical Signal Processing and Control, Elsevier, 10, 65-78. doi:10.1016/j.bspc.2013.12.009

[3] S.J. Rogers, L.A.Vismara. Evidence-based comprehensive treatments for early autism. J Clin Child Adolesc Psychol. 2008; 37(1):8 -38

[4] Le, H.H., Loomes, M.J. \& Loureiro, R.C.V., 2012. Mapping Arm Movements to Robotic Sonic Interaction Promote Group Dynamics and Increase Engagement at a Task. In International Conference on Neurorehabilitation. Toledo, Spain, pp. 843-846.

[5] Le, H.H.; Loureiro, R.C.V.; Dussopt, F.; Phillips, N.; Zivanovic, A; Loomes, M.J., "Soundscape and Haptic Cues in an Interactive Painting: a Study with Autistic Children," 2014 IEEE International Conference on Biomedical Robotics and Biomechatronics (BIOROB) August 2014 